\title{
Polarizable Poisson-Boltzmann equation: The study of polarizability effects on the structure of a double layer
}

Cite as: J. Chem. Phys. 134, 234704 (2011); https://doi.org/10.1063/1.3598476

Submitted: 04 March 2011 . Accepted: 18 May 2011 . Published Online: 16 June 2011

Derek Frydel

ARTICLES YOU MAY BE INTERESTED IN

Dipolar Poisson-Boltzmann approach to ionic solutions: A mean field and loop expansion analysis

The Journal of Chemical Physics 139, 164909 (2013); https://doi.org/10.1063/1.4826103

Effects of image charges on double layer structure and forces

The Journal of Chemical Physics 139, 124702 (2013); https://doi.org/10.1063/1.4821636

Self-consistent field model for strong electrostatic correlations and inhomogeneous dielectric media

The Journal of Chemical Physics 141, 244903 (2014); https://doi.org/10.1063/1.4904728

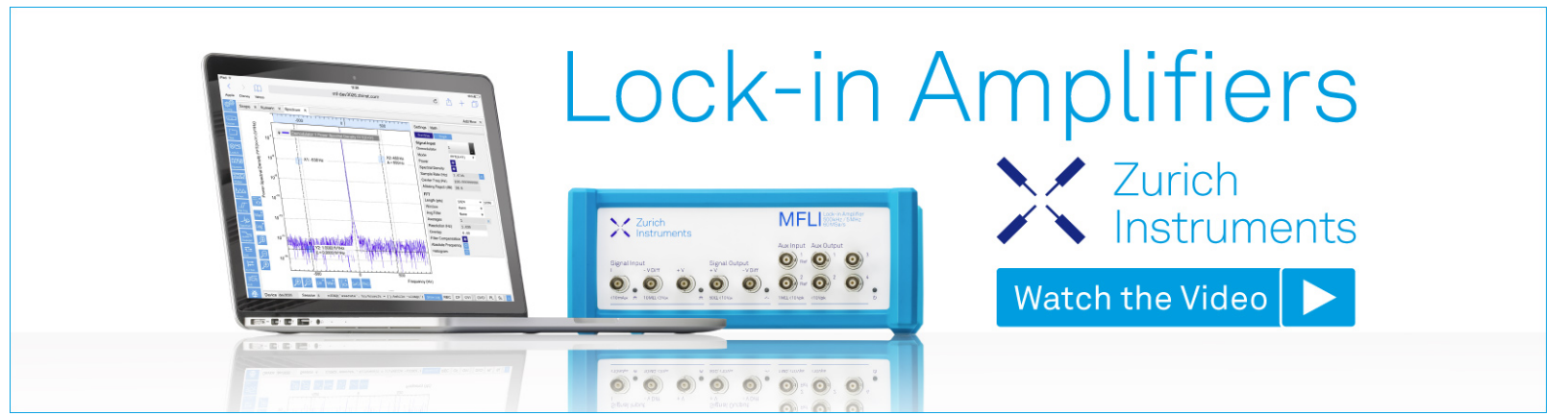

J. Chem. Phys. 134, 234704 (2011); https://doi.org/10.1063/1.3598476 


\title{
Polarizable Poisson-Boltzmann equation: The study of polarizability effects on the structure of a double layer
}

\author{
Derek Frydel ${ }^{\mathrm{a})}$ \\ Institute of Physics, The Federal University of Rio Grande do Sul, P.O. Box 15051, 91501-970 Porto Alegre, \\ RS, Brazil
}

(Received 4 March 2011; accepted 18 May 2011; published online 16 June 2011)

\begin{abstract}
We incorporate ion polarizabilities into the Poisson-Boltzmann equation by modifying the effective dielectric constant and the Boltzmann distribution of ions. The extent of the polarizability effects is controlled by two parameters, $\gamma_{1}$ and $\gamma_{2} ; \gamma_{1}$ determines the polarization effects in a dilute system and $\gamma_{2}$ regulates the dependence of the polarizability effects on the concentration of ions. For a polarizable ion in an aqueous solution $\gamma_{1} \approx 0.01$ and the polarizability effects are negligible. The conditions where $\gamma_{1}$ and/or $\gamma_{2}$ are large and the polarizability is relevant involve the low dielectric constant media, high surface charge, and/or large ionic concentrations. () 2011 American Institute of Physics. [doi:10.1063/1.3598476]
\end{abstract}

\section{INTRODUCTION}

At large separations ion interactions are dominated by the Coulomb forces and ions are efficiently represented as point charges (assuming the absence of permanent dipoles and higher order multipoles). The point charge representation is accurate as long as ions retain large separations. As separations decrease, electronic structure of ions and associated with its quantum effects, contribute additional interactions. At very small separations, an overlap of two electron clouds leads to strong repulsion. This can be represented either as an excluded volume effect or a soft repulsion (like the repulsive term $1 / r^{12}$ in the Lennard-Jones potential; the attractive term of this potential accounts for dissipation interactions). Another class of interactions deriving from quantum effects arises on account of the mobility of an electron cloud of an ion and is characterized by the frequency dependent polarizability, $\alpha(\omega)$. On the one hand, dynamic polarizability associated with high frequencies, determines the dispersion interactions as a result of high frequency correlated fluctuations of neighboring electron clouds, leading to attraction $1 / r^{6}$ (Ref. 1) (this algebraic form reflects the dipole-induced dipole interaction). On the other hand, low frequency fluctuations of an electrostatic field of thermally excited system induce "slowly" varying distortions of electron clouds, leading to induced interactions characterized by the static polarizability, $\alpha(0)$. Thus, a net charge of one ion induces a dipole moment in the neighboring ion, leading to attractive $1 / r^{4}$ potential. The presence of the charge-induced dipole attraction is evident in the formation of clusters of the like-charged ions. ${ }^{2}$ Quantum computations for two isolated ions $\mathrm{Na}^{+}$and $\mathrm{Cl}^{-}$show that an attraction at short separations comes from the charge-induced dipole interaction, while the dispersion term $1 / r^{6}$ constitutes a negligible correction. ${ }^{3}$

For a neutral air-water interface, static polarizability of ions plays a crucial role in determining an interface structure

\footnotetext{
a) Author to whom correspondence should be addressed. Electronic mail: dfrydel@gmail.com.
}

and its electrostatic properties. ${ }^{4}$ It is not polarizability per se that determines the structure, that is, not its interactions with a modified image charge alone. Rather it is an outcome of a delicate balance of interplays between ion polarizability and size, with surrounding water molecules, in particular an ability of an ion to form and cast away a hydration shell. Polarizability itself becomes important once a partially unhydrated ion crosses an interface..$^{5}$ As a result, soft ions show affinity for an interface and, in consequence, a neutral interface acquires charge.

Contributions of static polarizability are also observed in bulk solutions. Dielectric response of water decreases linearly with an electrolyte concentration (at concentrations $<0.2 \mathrm{M}$ ), and the dielectric decrement vary with the type of salt, roughly following the sequence of polarizability (or size) - the more polarizable (or larger) ions effect greater decrement. ${ }^{6,7}$ This behavior is attributed to the fact that solvent molecules trapped in a hydration sheath of an ion are less susceptible to an external electrostatic field. In effect, the number of free solvent molecules susceptible to polarization is reduced.

Furthermore, polarizability and size are found to regulate the adsorption of counterions in the double layer on a charged surface; large polarizable counterions are more efficient in penetrating the hydration layer adhering to a charged surface and bind directly with the surface charges. ${ }^{8-10}$ Once more, this behavior is mediated by solvent molecules.

In the present work, we investigate the polarizability contributions to electrostatics of the double layer by modifying the Poisson-Boltzmann (PB) equation, in the spirit of the models such as the modified PB equation which incorporates the finite size effects, ${ }^{11}$ the dipolar PB equation which incorporates a dipole structure, ${ }^{12-14}$ or its generalization to the higher order multipoles. ${ }^{15}$ In the polarizable PB equation developed in this work ions are represented as points which acquire an induced dipole moment when exposed to an external field. As each ion type is characterized by two parameters, charge and polarizability, the model permits to explore some 
aspects of ion specificity. In the model, a solvent is represented as a background dielectric constant $\epsilon$. We find that polarizability contributions are small for aqueous solutions, where $\epsilon$ is large and electrostatic interactions are screened but becomes important for low $\epsilon$. A condition of low $\epsilon$ is realized in ionic liquids where a solvent is altogether absent.

\section{PRELIMINARY CONSIDERATIONS}

We regard a dipole in terms of mechanics, as springs and charges; it is comprised of two charges, $q$ and $-q$, attached by a spring but not interacting with each other electrostatically (that is, the electrostatic self-energy of a dipole is not included). The dipole moment depends on the separation $\mathbf{s}$ between the charges, $\mathbf{p}=q \mathbf{s}$. The electrostatic force $q \mathbf{E}$ pulls the two charges apart and the separation $\mathbf{S}$ is regulated by the spring constant $k, q \mathbf{E}=k \mathbf{s}$. Relating the separation to the dipole moment we get $\mathbf{p}=\left(q^{2} / k\right) \mathbf{E}$, so that the static polarizability is inversely proportional to the spring constant $\alpha=q^{2} / k$.

The interaction potential between two ions with charge $Q_{1}$ and $Q_{2}$, and dipole moment $\mathbf{p}_{1}$ and $\mathbf{p}_{2}$ is

$$
\begin{aligned}
U(\mathbf{r})= & \frac{Q_{1} Q_{2}}{4 \pi \epsilon r}+\frac{Q_{1}\left(\mathbf{p}_{2} \cdot \mathbf{r}\right)-Q_{2}\left(\mathbf{p}_{1} \cdot \mathbf{r}\right)}{4 \pi \epsilon r^{3}} \\
& +\frac{\mathbf{p}_{1} \cdot \mathbf{p}_{2}}{4 \pi \epsilon r^{3}}-3 \frac{\left(\mathbf{p}_{1} \cdot \mathbf{r}\right)\left(\mathbf{p}_{2} \cdot \mathbf{r}\right)}{4 \pi \epsilon r^{5}},
\end{aligned}
$$

where $\mathbf{r}=\mathbf{r}_{1}-\mathbf{r}_{2}$, is the vector connecting the two particles, $r=\left|\mathbf{r}_{1}-\mathbf{r}_{2}\right|$, and $\epsilon$ is the dielectric constant of a medium. The terms are the charge-charge, charge-dipole, and dipoledipole interactions. A dipole that is induced (and not permanent) has the same orientation as the field that induces it. For the case of two isolated ions this implies $\mathbf{p}_{i}=p_{i}(\mathbf{r} / r)$. Furthermore, there is elastic energy cost associated with distortion of an electron could, $p_{i}^{2} / 2 \alpha_{i}$. The interaction potential between two polarizable ions with polarizability $\alpha_{1}$ and $\alpha_{2}$, is

$$
U(\mathbf{r})=\frac{Q_{1} Q_{2}}{4 \pi \epsilon r}-\frac{Q_{1} p_{2}+Q_{2} p_{1}}{4 \pi \epsilon r^{2}} \pm 2 \frac{p_{1} p_{2}}{4 \pi \epsilon r^{3}}+\frac{p_{1}^{2}}{2 \alpha_{1}}+\frac{p_{2}^{2}}{2 \alpha_{2}} .
$$

The dipole-dipole interaction is repulsive for the like-charged ions and attractive for opposite-charged ions. The chargedipole interaction is always attractive. The induced dipole moments, $p_{i}$, are obtained by minimizing the potential energy with respect to $p_{i}$. We find $p_{i}=\alpha_{i} Q_{j} / 4 \pi \epsilon r^{2}+O\left(1 / r^{5}\right)$, where $i \neq j$, and the attractive charge-induced dipole interaction between two polarizable ions is

$$
U_{\text {ind }}=-\frac{\left(Q_{1}^{2} \alpha_{2}+Q_{2}^{2} \alpha_{1}\right)}{2(4 \pi \epsilon)^{2}} \frac{1}{r^{4}}+O\left(1 / r^{7}\right) .
$$

The induced attraction competes with the Coulomb repulsion and for $Q_{1}=Q_{2}$ and $\alpha_{1}=\alpha_{2}$ the two like-charged ions start to attract at $r_{0}=(\alpha / \pi \epsilon)^{1 / 3}$. For example, for two iodide ions in an aqueous solution $r_{0}<1 \AA$. Such small separations are prevented by strongly overlapping electron clouds so this type of an interaction is irrelevant for aqueous solutions (see Ref. 16).

The induced interactions arise also between a charged macromolecule and a polarizable ion. For example, an infinite charged wall produces constant electrostatic field pro- portional to the surface charge, $\sigma_{c}$, which attracts or repels ions, depending on the charge sign, and, if an ion is polarizable, it induces a dipole moment. This dipole does not interact with the wall; there is no energy gain or loss as it changes its position. Its interactions with the wall must be mediated by other ions in the system. Thus, in the presence of counterions that screen the the surface charge, a single polarizable neutral molecule introduced into the system will drift towards the charged surface where an electrostatic field is least screened. To refine this thought experiment we take the screened potential at the charged wall of the Debye-Hückel theory, $\psi=\sigma_{c} e^{-\kappa x} / \sqrt{2 q^{2} c_{s} \beta \epsilon}$, where $\kappa^{-1}=\sqrt{\epsilon / 2 q^{2} c_{s} \beta}$ is the screening length, $\beta=k_{B} T, c_{s}$ is the bulk salt concentration, and $q$ is the ion charge (assumed to be the same for counterions and coions), and $\epsilon$ is the dielectric constant of a medium. The induced dipole moment of the polarizable molecule in this potential is $p=\alpha \sigma_{c} e^{-\kappa x} / \epsilon$. The resulting interaction potential between an induced dipole and the wall is

$$
\beta U_{\text {ind }}=-\frac{\beta \alpha \sigma_{c}^{2}}{2 \epsilon^{2}} e^{-2 \kappa x} .
$$

If the polarizable test particle in addition carries a charge - so that it represents a polarizable counterion - the total interaction with the wall is

$$
\beta U_{\mathrm{tot}}=-\sqrt{\left(\frac{\beta \sigma_{c}^{2}}{2 \epsilon}\right) \frac{e^{-2 \kappa x}}{c_{s}}}-\left(\frac{\beta \sigma_{c}^{2}}{2 \epsilon}\right) \frac{\alpha e^{-2 \kappa x}}{\epsilon} .
$$

The induced interaction is controlled by the dimensionless parameter $\beta \alpha \sigma_{c}^{2} / 2 \epsilon^{2}$. Furthermore, since the induced interaction has to be considered in relation to the competing charge interaction, polarizability effects are stronger when the dimensionless parameter $2 \epsilon c_{s} / \beta \sigma_{c}^{2}$ is large. This essentially points to the strongly correlated limit. Interesting to note is that the screening length of the induced interactions is twice as short as that for the charge interactions.

Finally, we note that when all counterions are polarizable, one has to consider that the induced by the charged surface dipoles will interact with each other, leading to the charge-dipole and dipole-dipole interactions (see Eq. (1)). It is not immediately clear how these interactions would effect the structure of the double layer.

\section{POLARIZABLE POISSON-BOLTZMANN (PPB) EQUATION}

The total charge density of ions with both a net charge and a dipole moment is

$$
\hat{\rho}_{c}(\mathbf{r})=\sum_{j=1}^{K} \sum_{i=1}^{N_{j}}\left[\left(q_{j}-\mathbf{p}_{i}^{(j)} \cdot \nabla\right) \delta\left(\mathbf{r}-\mathbf{R}_{i}^{(j)}\right)\right],
$$

and the dipole density reads

$$
\hat{\mathbf{P}}(\mathbf{r})=\sum_{j=1}^{K} \sum_{i=1}^{N_{j}} \mathbf{p}_{i}^{(j)} \delta\left(\mathbf{r}-\mathbf{R}_{i}^{(j)}\right)
$$

Ions are represented as points but their electronic structure is enriched via addition of a dipole moment, $\mathbf{p}_{i}^{(j)}$. There are $K$ ionic species where each species has $N_{j}$ ions with charge 
$q_{j}$. The total charge density can be differentiated into the free charge density due to net charges

$$
\hat{\rho}_{0}(\mathbf{r})=\sum_{j=1}^{K} \sum_{i=1}^{N_{j}} q_{j} \delta\left(\mathbf{r}-\mathbf{R}_{i}^{(j)}\right),
$$

and the bound charge density due to dipoles

$$
\hat{\rho}_{b}(\mathbf{r})=-\nabla \cdot\left[\sum_{j=1}^{K} \sum_{i=1}^{N_{j}} \mathbf{p}_{i}^{(j)} \delta\left(\mathbf{r}-\mathbf{R}_{i}^{(j)}\right)\right] .
$$

The distribution of charges gives rise to an electrostatic potential, $\psi$, according to the Poisson equation

$$
\epsilon \nabla^{2} \psi=-\rho_{0}+\nabla \cdot \mathbf{P},
$$

where $\rho_{0}=\left\langle\hat{\rho}_{0}\right\rangle$ and $\mathbf{P}=\langle\hat{\mathbf{P}}\rangle$ are the ensemble averaged quantities.

In the mean-field treatment, each ion is exposed to a mean "external" potential due to all ions in the system. If $w_{j}(\mathbf{r})$ is the mean potential that an ion of a species $j$, at location $\mathbf{r}$ feels on account of all charges in the system, then the resulting Boltzmann distribution is $n_{j}=c_{j} e^{-\beta w_{j}(\mathbf{r})}$ (in the course of the developing of the model the constant $c_{j}$ will turn out to be the bulk concentration of the ionic species $j$ ). The mean potential acting on a polarizable ion of a species $j$ at location $\mathbf{r}$ is

$$
w_{j}(\mathbf{r})=q_{j} \psi(\mathbf{r})-\mathbf{p}_{j} \cdot \mathbf{E}(\mathbf{r})+\frac{\left|\mathbf{p}_{j}\right|^{2}}{2 \alpha_{j}} .
$$

The term $\left|\mathbf{p}_{j}\right|^{2} / 2 \alpha_{j}$ is the elastic energy cost of distorting an electron cloud. An induced dipole is always aligned with a field and its magnitude is determined by the balance between the electrostatic and elastic force (the condition $\left.\partial w_{j} / \partial p_{j}=0\right)$. This results in a position dependent dipole moment $\mathbf{p}_{j}(\mathbf{r})=\alpha_{j} \mathbf{E}(\mathbf{r})$. The resulting mean potential and the number density is

$$
\begin{gathered}
w_{j}=q_{j} \psi-\frac{\alpha_{j}|\mathbf{E}|^{2}}{2}, \\
n_{j}=c_{j} e^{-\beta\left(q_{j} \psi-\alpha_{j}|\mathbf{E}|^{2} / 2\right)},
\end{gathered}
$$

respectively. Substituting the polarization and the free charge density,

$$
\begin{gathered}
\mathbf{P}(\mathbf{r})=\left(\sum_{j=1}^{K} \alpha_{j} c_{j} e^{-\beta\left(q_{j} \psi-\alpha_{j}|\mathbf{E}|^{2} / 2\right)}\right) \mathbf{E}, \\
\rho_{0}(\mathbf{r})=\sum_{j} q_{j} c_{j} e^{-\beta\left(q_{j} \psi-\alpha_{j}|\mathbf{E}|^{2} / 2\right)},
\end{gathered}
$$

into the Poisson equation (Eq. (3)), gives the polarizable Poisson-Boltzmann equation,

$$
\begin{gathered}
\nabla \cdot\left[\left(\epsilon+\sum_{j=1}^{K} \alpha_{j} c_{j} e^{-\beta\left(q_{j} \psi-\alpha_{j}|\mathbf{E}|^{2} / 2\right)}\right) \nabla \psi\right] \\
=-\sum_{j=1}^{K} q_{j} c_{j} e^{-\beta\left(q_{j} \psi-\alpha_{j}|\mathbf{E}|^{2} / 2\right)},
\end{gathered}
$$

where we represent the contributions of polarizability as an effective dielectric constant,

$$
\epsilon_{\mathrm{eff}}=\epsilon+\sum_{j=1}^{K} \alpha_{j} c_{j} e^{-\beta\left(q_{j} \psi-\alpha_{j}|\mathbf{E}|^{2} / 2\right)}
$$

The boundary conditions at the charged surface are

$$
\left(\epsilon+\sum_{j=1}^{K} \alpha_{j} c_{j} e^{-\beta\left(q_{j} \psi-\alpha_{j}|\mathbf{E}|^{2} / 2\right)}\right) \nabla \psi=-\sigma_{c},
$$

where $\sigma_{c}$ is the surface charge.

\section{A. Ions with permanent dipole moments}

Although the present work focuses on polarizable ions, it is instructive to consider ions with permanent dipoles, where $p_{j}$ represents the magnitude of a permanent dipole moment of a species $j$. Although a permanent dipole has a fixed magnitude, it retains an orientational degree of freedom,

$$
w_{j}(\mathbf{r}, \theta)=q_{j} \psi(\mathbf{r})-p_{j}|\mathbf{E}(\mathbf{r})| \cos \theta,
$$

where $\theta$ is the angle between a field and a dipole. This leads to the orientation dependent Boltzmann distribution, $\propto e^{-\beta\left(q_{j} \psi-p_{j}|\mathbf{E}| \cos \theta\right)}$. To obtain a distribution which depends on a position only, one needs to integrate the Boltzmann distribution over $\theta$,

$$
\begin{aligned}
n_{j} & =c_{j} e^{-\beta q_{j} \psi}\left(\frac{\int_{0}^{\pi} d \theta \sin \theta e^{\beta|\mathbf{E}| p_{j} \cos \theta}}{\int_{0}^{\pi} d \theta \sin \theta}\right) \\
& =c_{j} e^{-\beta q_{j} \psi}\left(\frac{\sinh \beta|\mathbf{E}| p_{j}}{\beta|\mathbf{E}| p_{j}}\right) .
\end{aligned}
$$

On average, the permanent dipole prefers orientation along an electrostatic field, therefore, through the dependence on the local field, an average dipole is a function of a position, $\mathbf{p}_{j}(\mathbf{r})=p_{j}\langle\cos \theta\rangle_{\theta} \mathbf{E} /|\mathbf{E}|$, where

$$
\begin{aligned}
\langle\cos \theta\rangle_{\theta} & =\frac{\int_{0}^{\pi} d \theta \sin \theta \cos \theta e^{\beta|\mathbf{E}| p_{j} \cos \theta}}{\int_{0}^{\pi} d \theta \sin \theta e^{\beta|\mathbf{E}| p_{j} \cos \theta}} \\
& =\operatorname{coth}\left(\beta|\mathbf{E}| p_{j}\right)-\frac{1}{\beta|\mathbf{E}| p_{j}}=\mathcal{L}\left(\beta|\mathbf{E}| p_{j}\right),
\end{aligned}
$$

and $\mathcal{L}\left(\beta|\mathbf{E}| p_{j}\right)$ is the Langevin function. The position dependent dipole is

$$
\mathbf{p}_{j}(\mathbf{r})=\frac{p_{j} \mathcal{L}\left(\beta|\mathbf{E}| p_{j}\right) \mathbf{E}}{|\mathbf{E}|}
$$

In the limit of small $\beta|\mathbf{E}| p_{j}, \mathbf{p}_{j}(\mathbf{r})=\frac{\beta p_{j}^{2}}{3} \mathbf{E}$, so it is linear in the local field, as for the case of polarized ions. In the limit of large $\beta|\mathbf{E}| p_{j}, \mathbf{p}_{j}(\mathbf{r})=\frac{p_{j} \mathbf{E}}{|\mathbf{E}|}$, and it saturates at its maximum possible magnitude $p_{j}$.

The resulting Poisson-Boltzmann type of an equation for ions with permanent dipoles is

$$
\nabla \cdot\left[\epsilon_{\mathrm{eff}} \nabla \psi\right]=-\sum_{j=1}^{K} q_{j} c_{j} e^{-\beta q_{j} \psi}\left(\frac{\sin \beta|\mathbf{E}| p_{j}}{\beta|\mathbf{E}| p_{j}}\right),
$$


where

$$
\epsilon_{\mathrm{eff}}=\epsilon+\sum_{j=1}^{K} p_{j} c_{j} e^{-\beta q_{j} \psi}\left(\frac{\sinh \beta|\mathbf{E}| p_{j}}{\beta|\mathbf{E}| p_{j}}\right) \frac{\mathcal{L}\left(\beta|\mathbf{E}| p_{j}\right)}{|\mathbf{E}|} .
$$

\section{B. Stress tensor}

The mean force acting on an ion of a species $j$ in the position $\mathbf{r}$ is $\mathbf{F}_{j}(\mathbf{r})=-\nabla w_{j}(\mathbf{r})$. Given the distribution of ions $n_{j}$, the force density is $\mathbf{f}_{j}(\mathbf{r})=-n_{j} \nabla w_{j}(\mathbf{r})$. Combining contributions of all ionic species, for polarizable ions we have

$$
\mathbf{f}=\sum_{j=1}^{K}\left(q_{j} n_{j} \mathbf{E}+\frac{\alpha_{j}}{2} n_{j} \nabla E^{2}\right) .
$$

In equilibrium, the electrostatic force is counterbalanced by the osmotic force and the total force density is ${ }^{17}$

$$
\mathbf{f}_{\mathrm{tot}}=\sum_{j=1}^{K}\left(q_{j} n_{j} \mathbf{E}+\frac{\alpha_{j}}{2} n_{j} \nabla E^{2}-k_{B} T \nabla n_{j}\right)=0 .
$$

Using the PPB equation together with the identity $\mathbf{E}(\nabla \cdot \mathbf{E})$ $=\nabla \cdot(\mathbf{E} \otimes \mathbf{E})-\frac{1}{2} \nabla E^{2}$ we rewrite the force density in the form $\mathbf{f}_{\text {tot }}=\nabla \cdot \mathbf{T}$. The resulting stress tensor is

$$
\mathbf{T}=\left(\epsilon+\sum_{j} \alpha_{j} n_{j}\right) \mathbf{E} \otimes \mathbf{E}-\left(\frac{\epsilon}{2} E^{2}+\Pi\right) \mathbf{I} .
$$

The osmotic pressure is $\Pi=k_{B} T \sum_{j}\left(n_{j}-c_{j}\right)$ and $n_{j}$ is given in Eq. (4).

\section{RESULTS}

In the following, we study a symmetric $1: 1$ electrolyte for a charged wall at $x=0$ with an electrolyte confined to the half-space $x \geq 0$. Solvent is represented as a background dielectric constant $\epsilon$ and the polarizability of all ions is set to $\alpha$. The PPB equation for a described system reads

$$
\begin{aligned}
& \frac{d}{d x}\left[\left(\epsilon+2 \alpha c_{s} \cosh (e \beta \psi) e^{\beta \alpha \psi^{\prime 2} / 2}\right) \psi^{\prime}\right] \\
& \quad=2 e c_{s} \sinh (e \beta \psi) e^{\beta \alpha \psi^{\prime 2} / 2},
\end{aligned}
$$

where $e$ is the elementary charge, $c_{s}$ is the bulk density of ions, $\epsilon$ is the medium dielectric constant, and $\psi^{\prime}=\frac{d \psi}{d x}$. The boundary conditions at the charged wall are

$$
\left[\epsilon+2 \alpha c_{s} \cosh (e \beta \psi) e^{\beta \alpha \psi^{\prime 2} / 2}\right] \psi^{\prime}=-\sigma_{c},
$$

where $\sigma_{c}>0$ and an effective dielectric constant is

$$
\epsilon_{\mathrm{eff}}=\epsilon+2 \alpha c_{s} \cosh (e \beta \psi) e^{\beta \alpha \psi^{\prime 2} / 2} .
$$

The quantum chemical calculations give the following polarizabilities of halides in the gas phase: $\bar{\alpha}\left(\mathrm{F}^{-}\right)=2.47 \AA^{3}$, $\bar{\alpha}\left(\mathrm{Cl}^{-}\right)=5.48 \AA^{3}, \quad \bar{\alpha}\left(\mathrm{Br}^{-}\right)=7.27 \AA^{3}, \quad \bar{\alpha}\left(\mathrm{I}^{-}\right)=10.27 \AA^{3} .{ }^{18}$ Polarizabilities are reduced in an aqueous solution: $\bar{\alpha}\left(\mathrm{F}^{-}\right)=1.3 \AA^{3}, \quad \bar{\alpha}\left(\mathrm{Cl}^{-}\right)=3.5 \AA^{3}, \quad \bar{\alpha}\left(\mathrm{Br}^{-}\right)=4.6 \AA^{3}, \quad$ and $\bar{\alpha}\left(\mathrm{I}^{-}\right)=7.5 \AA^{3}{ }^{19} \bar{\alpha}=\alpha / 4 \pi \epsilon_{0}$ where $\epsilon_{0}$ is the dielectric constant of a vacuum. If we consider the dimensionless

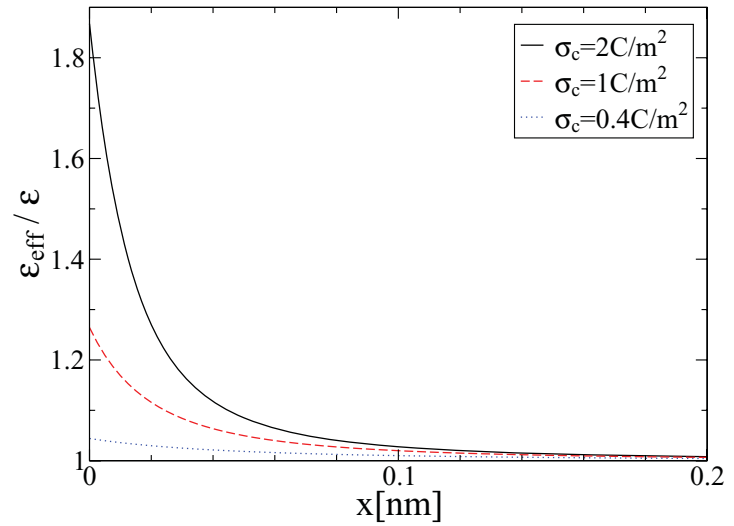

FIG. 1. Effective dielectric constant, Eq. (13), for different $\sigma_{c}$. The system parameters are $c_{s}=10^{-5} \mathrm{M}$ (corresponding to the screening length $100 \mathrm{~nm}$ ), $\bar{\alpha}=\alpha / 4 \pi \epsilon_{0}=10 \AA^{3}$, and $\epsilon / \epsilon_{0}=80$, where $\epsilon_{0}$ is the dielectric constant in a vacuum.

polarizability parameter, $\beta \alpha \sigma_{c}^{2} / 2 \epsilon^{2}$, obtained for the DebyeHückel potential (Eq. (2)), the polarizability effects in electrolyte can be expected to be small. This parameter for an iodine ion near a charged colloidal surface gives $\approx 0.03$. By reducing the dielectric constant from its value inside water $\left(\epsilon=80 \epsilon_{0}\right)$ to the value in vacuum $\left(\epsilon=\epsilon_{0}\right)$, this parameter increases to $\approx 250$. This suggests an important role of polarizability in ionic liquids where no solvent is present.

We consider electrolyte solutions first. Figure 1 shows profiles of an effective dielectric constant, Eq. (13), resulting from accumulation of soft counterions, with $\bar{\alpha}=10 \AA^{3}$, against a charged wall. $\epsilon$ is set to the value of water $\epsilon / \epsilon_{0}=80$. $\epsilon_{\text {eff }}$ follows the distribution of counterions, $\epsilon_{\text {eff }} \approx \epsilon+\alpha n_{-}$. A typical colloidal surface charge spans the range $\sigma_{c} \in[0.1,0.4]\left\{\mathrm{C} / \mathrm{m}^{2}\right\}$, depending on size and material. For electrolytes, thus, polarizability is relevant for superficially large surface charges. Still, it is worthwhile to discuss the observed trends. Examining the PPB equation it is not immediately clear how polarizability should redistribute the counterion cloud; on the one hand, the term $-\alpha \psi^{\prime 2} / 2$ in the Boltzmann distribution suggests enhancement of a density; on the other hand, the increase of $\epsilon_{\text {eff }}$ implies weaker electrostatic effects. The results in Fig. 2 show that the

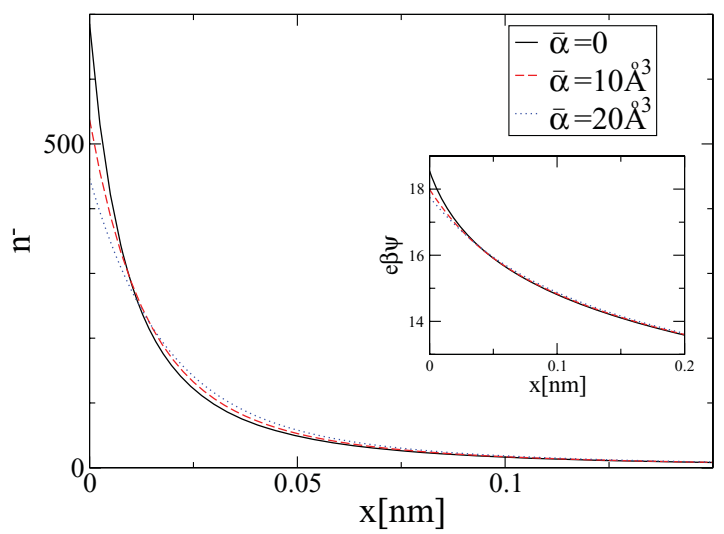

FIG. 2. The number density of counterions, $n^{-}=c_{s} e^{\beta\left(e \psi+\alpha \psi^{\prime 2} / 2\right)}$, in units $\mathrm{nm}^{-3}$. The system parameters are $c_{s}=10^{-5} \mathrm{M}, \sigma_{c}=2 \mathrm{C} / \mathrm{m}^{2}$, and $\epsilon / \epsilon_{0}$ $=80$. 
counterion cloud becomes more diffuse. A similar "spreading out" effect is seen in the electrostatic potential and field.

A different way to analyze the effects of polarizability is according to the equation $\epsilon \nabla^{2} \psi=-\rho_{0}+\nabla \cdot \mathbf{P}$, where the polarizability contributions are described in terms of the bound density $\rho_{b}=-\nabla \cdot \mathbf{P}$, instead of $\epsilon_{\text {eff }}$. Accordingly we rewrite the PPB equation for the present wall model,

$$
\begin{aligned}
\epsilon \psi^{\prime \prime}= & 2 e c_{s} \sinh (e \beta \psi) e^{\beta \alpha \psi^{\prime 2} / 2} \\
& -2 c_{s} \alpha \frac{d}{d x}\left[\psi^{\prime} \cosh (e \beta \psi) e^{\beta \alpha \psi^{\prime 2} / 2}\right] .
\end{aligned}
$$

The boundary conditions in this interpretation read

$$
\epsilon \psi^{\prime}=-\left(\sigma_{c}+\sigma_{p}\right),
$$

where $\sigma_{p}=\hat{\mathbf{n}} \cdot \mathbf{P}$ is the surface polarization density and $\hat{\mathbf{n}}$ is a normal to the surface pointing away from an electrolyte. For the present model

$$
\sigma_{p}=2 c_{s} \alpha \psi_{c}^{\prime} \cosh \left(e \beta \psi_{c}\right) e^{\beta \alpha \psi_{c}^{\prime 2} / 2},
$$

where the subscript $c$ indicates the contact value (value at the charged surface),

$$
\begin{array}{r}
\rho_{0}=-2 e c_{s} \sinh (e \beta \psi) e^{\beta \alpha \psi^{\prime 2} / 2}, \\
\rho_{b}=2 c_{s} \alpha \frac{d}{d x}\left[\psi^{\prime} \cosh (e \beta \psi) e^{\beta \alpha \psi^{\prime 2} / 2}\right] .
\end{array}
$$

The two conditions of electroneutrality are

$$
\int d \mathbf{r} \rho_{0}(\mathbf{r})=-\frac{\sigma_{c}}{\epsilon},
$$

for the bare surface charge and

$$
\int d \mathbf{r} \rho_{b}(\mathbf{r})=-\frac{\sigma_{p}}{\epsilon}
$$

for the polarization surface charge. Figure 3 shows the free and bound charge densities for two different values of $\alpha$. Both charge densities diffuse with increasing $\alpha$, and, while the number of free charges is constant, the number of bound charges increases with increasing $\alpha$.

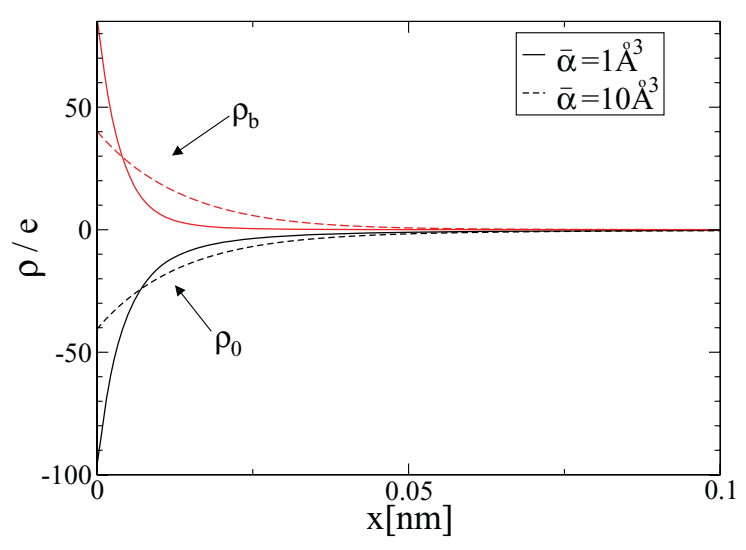

FIG. 3. The free and bound charge density distributions, $\rho_{0} e^{-1}$ and $\rho_{b} e^{-1}$, respectively (see Eqs. (16) and (17)) in units $\mathrm{nm}^{-3}(e$ is the elementary charge). The system parameters are $c_{s}=0.1 \mathrm{M}, \sigma_{c}=0.1 \mathrm{C} / \mathrm{m}^{2}$, and $\epsilon$ $/ \epsilon_{0}=1$.

\section{A. The contact value theorem}

The properties at the contact with the wall can be quantified using the contact value theorem, which can be derived from the stress tensor $\mathbf{T}$ in Eq. (10). We recall that the force acting on a body enclosed by a surface $\partial \Omega$ is

$$
\mathbf{f}=\oint_{\partial \Omega} d S \hat{\mathbf{n}} \cdot \mathbf{T}
$$

For a single wall, the force exerted on the wall (at $x=0$ ) vanishes,

$$
f_{x}(0)=\int d A T_{x x}(0)=0
$$

where

$$
T_{x x}(0)=\frac{\epsilon}{2} \psi_{c}^{\prime 2}+P_{c} \psi_{c}^{\prime}-k_{B} T\left(n_{c}^{+}+n_{c}^{-}-2 c_{s}\right),
$$

is the $x x$ tensor component calculated at the wall and the subscript $c$ denotes the contact value of a function. Using the boundary conditions in Eq. (15) and the equality $\sigma_{p}=-P_{c}$ we arrive at the contact value theorem,

$$
\frac{\beta}{2 \epsilon}\left(\sigma_{c}^{2}-\sigma_{p}^{2}\right)=n_{c}^{-}+n_{c}^{+}-2 c_{s} .
$$

We can eliminate the dependence on density by using the relation $\sigma_{p}=\alpha\left(n_{c}^{-}+n_{c}^{+}\right) \psi_{c}^{\prime}$ together with the boundary conditions. The result is the cubic equation for the ratio $\sigma_{p} / \sigma_{c}$,

$$
\left(\frac{\sigma_{p}}{\sigma_{c}}\right)^{3}+\left(\frac{\sigma_{p}}{\sigma_{c}}\right)^{2}-\left(\frac{\sigma_{p}}{\sigma_{c}}\right)\left(1+\gamma_{2}+\frac{1}{\gamma_{1}}\right)-\left(1+\gamma_{2}\right)=0
$$

where the two dimensionless parameters controlling the behavior of $\sigma_{p} / \sigma_{c}$ are $\gamma_{1}=\beta \alpha \sigma_{c}^{2} / 2 \epsilon^{2}$ and $\gamma_{2}=4 c_{s} \epsilon / \beta \sigma_{c}^{2}$. (The same parameters emerge in the simple Debye-Hückel model of Eq. (2).) For $\gamma_{1}, \gamma_{2}>0$, only one real root exists. In the limit of small $\gamma_{1}, \sigma_{p} / \sigma_{c}$ $\approx-\left(\gamma_{1}\left(1+\gamma_{2}\right)\right) /\left(1+\gamma_{1}\left(1+\gamma_{2}\right)\right)$. In the limit $\gamma_{1} \rightarrow \infty$, $\sigma_{p}$ approaches $-\sigma_{c}$ from above such as $\sigma_{p} / \sigma_{c} \approx-1$ $+\left(1 /\left(1+\gamma_{1} \gamma_{2}\right)\right)$ - thus in highly concentrated solutions (such as ionic liquids) the polarizability is more relevant (see Fig. 4). In the limit $\alpha \rightarrow 0$, Eq. (19) reduces to the

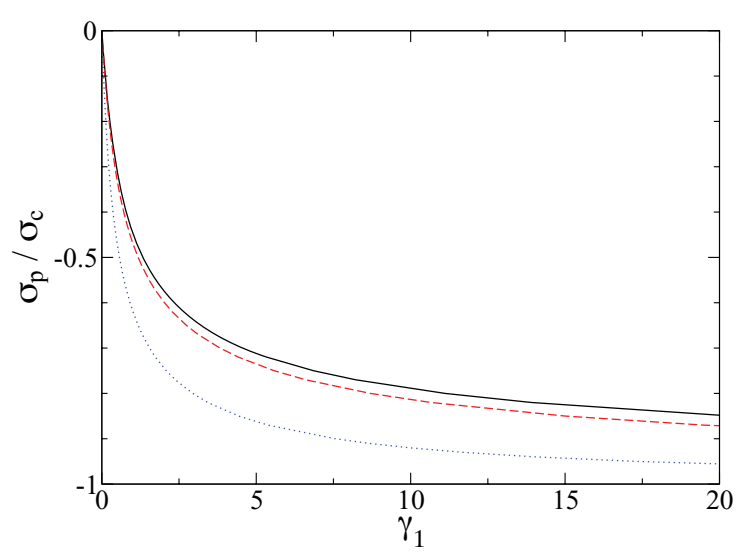

FIG. 4. The ratio $\sigma_{p} / \sigma_{c}$ as a function of $\gamma_{1}$. The solid, dashed, and dotted line is for $\gamma_{2}=0, \gamma_{2}=0.1$, and $\gamma_{2}=1$, respectively. For aqueous solution with $\sigma_{c}=0.1 \mathrm{C} / \mathrm{m}^{2}$ and $c_{s}=0.1 \mathrm{M}, \gamma_{2} \approx 0.1$. 


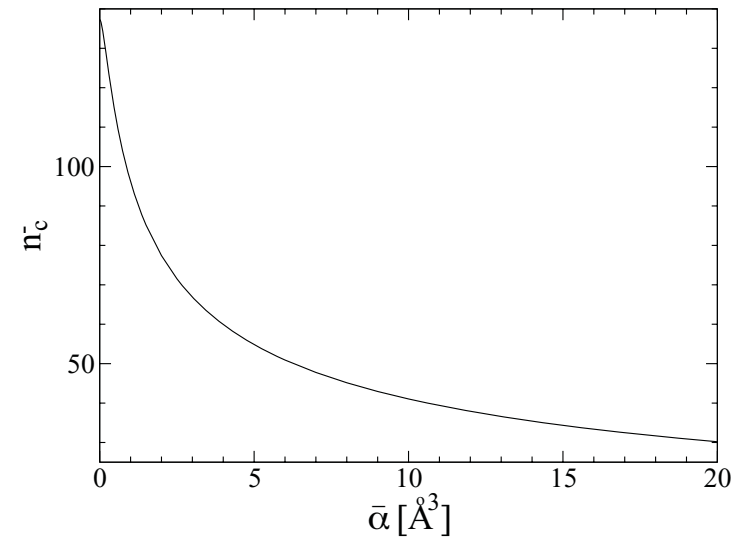

FIG. 5. The counterion density at the contact with the wall, $n_{c}^{-}=c_{s}$ $e^{\beta\left(e \psi_{c}+\alpha \psi_{c}^{\prime 2} / 2\right)}$ (in units $\mathrm{nm}^{-3}$ ), as a function of $\alpha$. The system parameters are $c_{s}=0.1 \mathrm{M}, \sigma_{c}=0.1 \mathrm{C} / \mathrm{m}^{2}$, and $\epsilon / \epsilon_{0}=1$.

standard contact value theorem of the PB equation,

$$
\frac{\beta \sigma_{c}^{2}}{2 \epsilon}=-\left(\frac{\sigma_{p}}{\sigma_{c}}\right) \frac{\epsilon}{\alpha}-2 c_{s}=n_{c}^{-}+n_{c}^{+}-2 c_{s} .
$$

It is possible to express the contact density in terms of the ratio $\sigma_{p} / \sigma_{c}$,

$$
\left(n_{c}^{-}+n_{c}^{+}\right)=-\frac{\epsilon}{\alpha}\left(\frac{\sigma_{p} / \sigma_{c}}{1-\sigma_{p} / \sigma_{c}}\right)
$$

and the similar expression can be obtained for the contact potential $\psi_{c}$. In Figs. 5 and 6 , we plot the counterion density and potential at the wall contact as a function of $\alpha$. As $\alpha$ $\rightarrow \infty$, electrostatic potential and field tend to zero and $\alpha \psi^{\prime} \rightarrow \frac{\sigma_{c}}{2 c_{s}}$.

\section{B. The far field behavior}

We have considered the influence of polarizability in the region near and at the charged wall. Another limit of interest is the far field domain. In this limit the electrostatic potential is weak, so that the background dielectric constant approaches the bulk value,

$$
\epsilon_{\text {bulk }}=\epsilon+2 c_{s} \alpha
$$

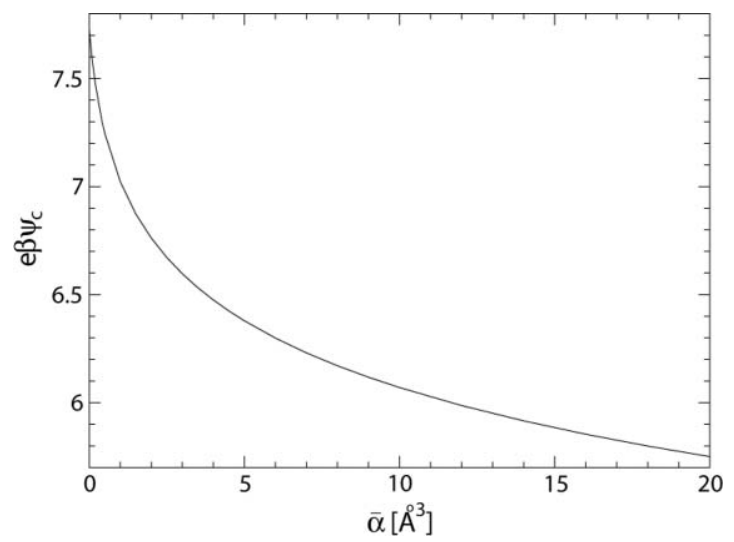

FIG. 6. The contact potential as a function of $\alpha$. The system parameters are $c_{s}=0.1 \mathrm{M}, \sigma_{c}=0.1 \mathrm{C} / \mathrm{m}^{2}$, and $\epsilon / \epsilon_{0}=1$.

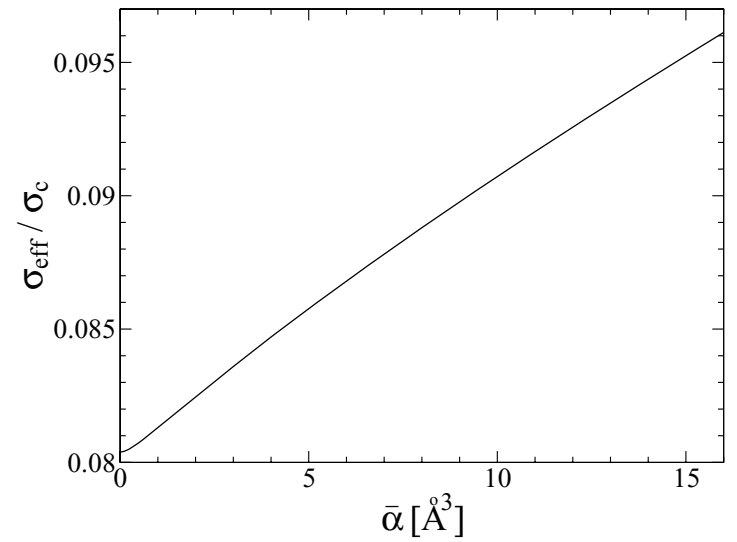

FIG. 7. The renormalized charge $\sigma_{\text {eff }}$ (obtained by fitting the potential far away from the wall to the form in Eq. (20)) as a function of $\alpha$. The parameters are $c_{s}=0.1 \mathrm{M}, \sigma_{c}=0.1 \mathrm{C} / \mathrm{m}^{2}$, and $\epsilon / \epsilon_{0}=1$.

while the potential itself decays according to the DebyeHückel functional form,

$$
\psi=\frac{\sigma_{\text {eff }}}{\sqrt{2 \beta e^{2} c_{s} \epsilon_{\text {bulk }}}} e^{-x \kappa},
$$

where $\kappa^{-1}=\sqrt{\epsilon_{\text {bulk }} / 2 e^{2} c_{s} \beta}$ is the screening length and $\sigma_{\text {eff }}$ is the renormalized surface charge determined by fitting the far field potential to the form in Eq. (20). The charge renormalization is attributed to the "condensation" of counterions at a charged surface when the attraction between the surface charges and counterions becomes larger than the competing thermal energy. The condensed counterions together with the bare surface charges constitute an effective surface charge. Figure 7 shows that polarizability increases the value of $\sigma_{\text {eff }}$. This can be traced to the reduced "condensation" of counterions with increased polarization as seen in Fig. 5.

\section{Other applications of the PPB equation}

To show off some of the applications of the PPB model, we investigate some aspects of ion specificity. We consider the case where half of the counterions is polarizable and the other half nonpolarizable: $n_{-}=\frac{1}{2} c_{s} e^{\beta e \psi}\left(1+e^{\beta \alpha \psi^{\prime 2} / 2}\right)$. (All coions are nonpolarizable.) As shown in Fig. 8 the polarizable counterions show affinity for the charged surface. The other case we consider is when all ions are nonpolarizable, but a third species of neutral but polarizable particles is introduced, $n_{-}=c_{s} e^{\beta \alpha \psi^{\prime 2} / 2}$. The total system is electroneutral where the surface charge is neutralized by counterions. These neutral particles are attracted to the charged surface as seen in Fig. 9.

\section{The nonlinear solvent effects}

As indicated by the dimensionless polarizability parameter $\gamma_{1}$, the polarizability effects are relevant for low dielectric constants. For electrolytes where $\epsilon / \epsilon_{0}=80$ polarizability acquires relevance for superficially large surface charges (see Fig. 2). At large surface charges, however, one has to 


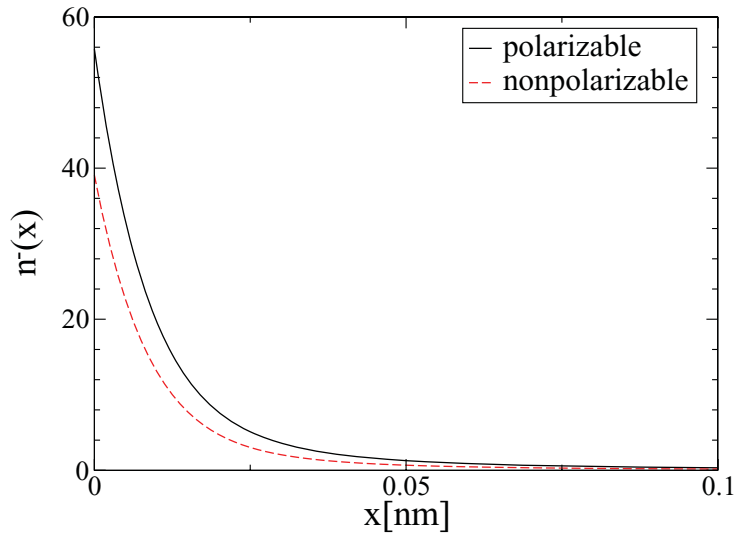

FIG. 8. The density profiles for polarizable, $\frac{1}{2} c_{s} e^{\beta\left(e \psi+\alpha \psi^{\prime 2} / 2\right)}$, and nonpolarizable, $\frac{1}{2} c_{s} e^{\beta e \psi}$, counterions (in units $\mathrm{nm}^{-3}$ ). The polarizability constant is $\bar{\alpha}=10 \AA^{3}$. All coions are nonpolarizable. Other parameters are $c_{s}=0.1 \mathrm{M}$, $\sigma_{c}=0.1 \mathrm{C} / \mathrm{m}^{2}$, and $\epsilon / \epsilon_{0}=1$.

take into account the nonlinear effects of solvent polarization which lead to polarization saturation and associated with it dielectric decrement. Saturation of polarization is characteristic of molecules with permanent dipoles, such as water solvent. The permanent dipoles in the external field behave according to Eq. (8): if electrostatic energy of a dipole in an external field dominates thermal energy, no more alignment of a dipole with a field is possible and the dipole moment saturates. The saturation of a water dipole should already be effective for $\sigma_{c} \approx 0.1 \mathrm{C} / \mathrm{m}^{2}$.

To account for the saturation of a solvent polarization we resort to the dipolar Poisson-Boltzmann equation. ${ }^{12}$ In this model the solvent molecules are represented as point dipoles. The resulting field dependent dielectric constant is

$$
\epsilon(|\mathbf{E}|)=p_{0} n_{d}\left(\beta p_{0}|\mathbf{E}|\right) \mathcal{L}\left(\beta p_{0}|\mathbf{E}|\right),
$$

where $p_{0}$ is the permanent dipole moment, $n_{d}$ is the solvent field dependent density, and $\mathcal{L}(x)=\operatorname{coth} x-1 / x$ is the Langevin function. For large surface charges, the dominant nonlinear effects should come from orientation saturation.

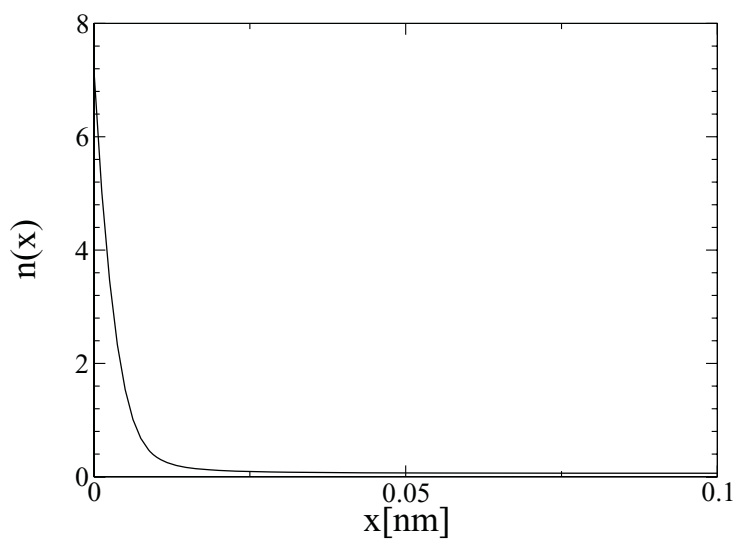

FIG. 9. The density profile of neutral polarizable particles, $n_{-}=c_{s} e^{\beta \alpha \psi^{\prime 2} / 2}$ (in units $\mathrm{nm}^{-3}$ ) with $\bar{\alpha}=10 \AA^{3}$. In addition, the system contains counterions and coions. The bulk density of each species is $c_{s}=0.1 \mathrm{M}$. The remaining parameters are $\sigma_{c}=0.1 \mathrm{C} / \mathrm{m}^{2}$ and $\epsilon / \epsilon_{0}=1$.

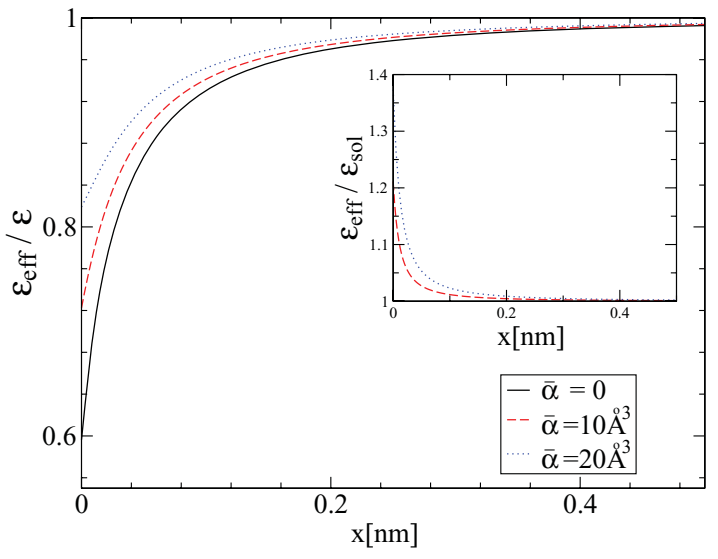

FIG. 10. Effective dielectric constant for a nonlinear solvent for different values of $\alpha$. The system parameters are $\sigma_{c}=0.4 \mathrm{C} / \mathrm{m}^{2}$ and $c_{s}=10^{-5} \mathrm{M}$. The inset shows $\epsilon_{\text {eff }}$ for different polarizabilities in relation to $\epsilon_{\mathrm{sol}}=\epsilon_{\mathrm{eff}}(\alpha=$ $0)$.

Neglecting compressibility effects, ${ }^{14}$ the total effective dielectric constant, which includes polarizability effects, is

$$
\epsilon_{\mathrm{eff}}=\epsilon_{0}+\frac{p_{0} c_{d} \mathcal{L}\left(\beta p_{0}|\mathbf{E}|\right)}{|\mathbf{E}|}+\alpha\left(n_{-}+n_{+}\right),
$$

and the Poisson-Boltzmann type of an equation for a charged wall is

$$
\begin{aligned}
& \frac{d}{d x}\left[\left(\epsilon_{0}+\frac{p_{0} c_{d} \mathcal{L}\left(\beta p_{0} \psi^{\prime}\right)}{\psi^{\prime}}+2 \alpha c_{s} \cosh (e \beta \psi) e^{\beta \alpha \psi^{\prime 2} / 2}\right) \psi^{\prime}\right] \\
& \quad=2 e c_{s} \sinh (e \beta \psi) e^{\beta \alpha \psi^{\prime 2} / 2} .
\end{aligned}
$$

The boundary conditions at the wall are

$$
\left[\epsilon_{0}+\frac{p_{0} c_{d} \mathcal{L}\left(\beta p_{0} \psi^{\prime}\right)}{\psi^{\prime}}+\alpha c_{s} e^{\beta\left(e \psi+\alpha \psi^{\prime 2} / 2\right)}\right] \psi^{\prime}=-\sigma_{c} .
$$

We set $c_{d}$ to the value of water, $c_{d}=55 \mathrm{M}$ and set $p_{0}=4.8 \mathrm{D}$ so that $\epsilon_{\text {eff }}(E \rightarrow 0) / \epsilon_{0} \approx 80$. (For liquid water $p_{0} \approx 3 \mathrm{D}$ ).

In Fig. 10 we show $\epsilon_{\text {eff }}$ for different values of ion polarizabilities. First, we bring attention to the case $\alpha=0$, where the nonlinear solvent effects lead to a dielectric decrement near a charged wall. The dielectric decrement is countered by the dielectric increment caused by polarizable ions (clearly seen in the inset of Fig. 10). Comparison with the results of Fig. 1 shows that the nonlinear solvent effects enhance the polarizability contributions. Yet despite the enhanced effect of polarizability on $\epsilon_{\text {eff }}$, the density and potential profiles (not shown) remain almost unaffected. Based on the present model, we conclude that polarizability is unimportant for aqueous solutions - even with the dielectric decrement taken into account.

In Fig. 11 we plot the polarization surface charge density due to separate contributions,

$$
\begin{gathered}
\sigma_{p}^{\mathrm{sol}}=p_{0} c_{d} \mathcal{L}\left(\beta p_{0} \psi_{c}^{\prime}\right), \\
\sigma_{p}^{\text {ion }}=2 \alpha c_{s} \psi_{c}^{\prime} \cosh \left(e \beta \psi_{c}\right) e^{\beta \alpha \psi_{c}^{\prime 2} / 2},
\end{gathered}
$$

of the solvent and polarizable ions, respectively. For the linear solvent, represented as a constant background dielectric 


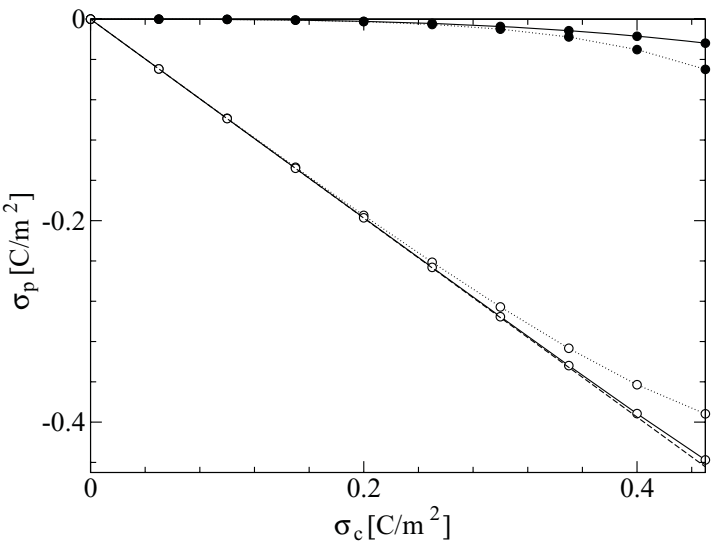

FIG. 11. $\sigma_{p}$ as a function of $\sigma_{c}$. The white and black symbols represent $\sigma_{p}^{\text {sol }}$ and $\sigma_{p}^{\text {ion }}$, respectively (Eqs. (21) and (22)). The dotted lines are for the case when both the nonlinear solvent and ion polarizability effects are included in the model. The solid lines are for the model with a single contribution. The dashed line is the result for the linear solvent (Eq. (23)). The system parameters are $c_{s}=10^{-5} \mathrm{M}$ and $\bar{\alpha}=10 \AA^{3}$.

constant,

$$
\sigma_{p}^{\mathrm{sol}}=\left(\frac{\epsilon-\epsilon_{0}}{\epsilon}\right) \sigma_{c}
$$

We consider $\sigma_{p}^{\text {sol }}$ at $\alpha=0$ first and compare the linear and nonlinear results. As seen in Fig. 11, the nonlinear contributions are rather weak and become noticeable for $\sigma_{c}$ $>0.4 \mathrm{C} / \mathrm{m}^{2}$. If we allow ions to be polarizable (the dotted line with white symbols), the nonlinear effects become more pronounced. The plots for $\sigma_{p}^{\text {ion }}$ show enhanced effect of polarizability with inclusion of the dielectric decrement effect (solid versus dotted lines with black symbols).

In the present model, we consider the dielectric decrement caused by a field of a highly charged surface. Another source of dielectric decrement results from the interactions between ions and solvent molecules: dipole moments of solvent molecules forming a hydration shell around an ion saturate in a strong field of an ion. ${ }^{7}$ Our model does not treat this effect. It is not clear how the two effects would compete and what an overall polarization would be. However, it is doubtful that this additional effect would be sufficiently strong to alter the conclusions of the present model.

\section{CONCLUSION}

In this work, we incorporate ion polarizabilities into the Poisson-Boltzmann equation by modifying the effective dielectric constant and the Boltzmann distribution of polarizable ions. From the contact value theorem we obtain the ratio $\sigma_{p} / \sigma_{c}$ as a function of two dimensionless parameters, $\gamma_{1}$ and $\gamma_{2}$. Increase of either of the parameters increases the polarizability effects. Based on this result we are able to determine the conditions where polarizability is relevant. For example, for an iodide ion in an aqueous solution, $\gamma_{1} \approx 0.01$, and the polarizability contributions are negligible. $\gamma_{1}$ becomes large for the low dielectric constant media and/or very high surfaces charges. The parameter $\gamma_{2}$, on the other hand, increases proportionally with the ion concentration. The low dielectric constant and high concentration conditions point to ionic liquids as a system where polarizability should not be neglected. The absence of a solvent in these systems, on the one hand, drastically increases the background dielectric constant, on the other hand, decreases inter-ionic separations. One must, however, be wary of the mean-field predictions at high densities where Coulomb correlations and finite size effects ${ }^{20}$ are extremely important.

The key conclusion of this work is primarily negative: polarizability has little relevance for typical aqueous solutions. By quantifying our results we are able to point to the conditions where such effects can play a relevant role. These conditions, however, are in the domain where the mean-field theory starts to break down. To properly investigate the polarizability contributions under such conditions, one needs to resort to more trustworthy theoretical descriptions. Despite its shortcomings, the present work constitutes a good and systematic starting point for studying systems with polarizable ions.

\section{ACKNOWLEDGMENTS}

The author is extremely grateful to Haim Diamant for unlimited access to computer resources. The author acknowledges useful discussions with Martin Oettel, Yan Levin, David Andelman, and Dan Ben-Yaakov. This work was supported by the German Research Foundation (DFG) through the Collaborative Research Centre SFB-TR6 ("Colloids in External Fields"), project N01 (Junior Research Group "Colloids at Fluid Interfaces"), and by the CNPq.

${ }^{1}$ F. London, Trans. Faraday Soc. 33, 8 (1937).

${ }^{2}$ A. M. Sapse, M. T. Rayez-Meaume, J. C. Rayez, and L. J. Massa, Nature (London) 278, 332 (1979).

${ }^{3}$ R. R. Netz, J. Phys.: Condens. Matter 16, S2353 (2004); Curr. Opin. Colloid Interface Sci. 9, 192 (2004).

${ }^{4}$ P. Jungwirth and D. J. Tobias, J. Phys. Chem. B 106, 6361 (2002).

${ }^{5}$ Y. Levin, Phys. Rev. Lett. 102, 147803 (2009).

${ }^{6}$ E. Glueckauf, Trans. Faraday Soc. 60, 1637 (1964).

${ }^{7}$ D. Ben-Yaakov, D. Andelman, and R. Podgornik, J. Chem. Phys. 134, 074705 (2011).

${ }^{8}$ C. D. Lorenz and A. Travesset, Phys. Rev. E 75, 061202 (2007).

${ }^{9}$ A. P. dos Santos and Y. Levin, Phys. Rev. Lett 106, 167801 (2011).

${ }^{10}$ D. Horinek and R. R. Netz, Phys. Rev. Lett. 99, 226104 (2007).

${ }^{11}$ I. Borhukov, D. Andelman, and H. Orland, Phys. Rev. Lett. 79, 435 (1997).

${ }^{12}$ A. Abrashkin, D. Andelman, and H. Orland, Phys. Rev. Lett. 99, 077801 (2007).

${ }^{13}$ C. Azuara, H. Orland, M. Bon, P. Koehl, and M. Delarue, Biophys. J. 95, 5587 (2008).

${ }^{14}$ D. Frydel and M. Oettel, Phys. Chem. Chem. Phys. 13, 4109 (2011).

${ }^{15}$ M. Kanduc, A. Naji, Y. S. Jho, P. A. Pincus, and R. Podgornik, J. Phys.: Condens. Matter 21, 424103 (2009).

${ }^{16} \mathrm{G}$. Ahn-Ercan, H. Krienke, and W. Kunz, Curr. Opin. Colloid Interface Sci. 9, 92 (2004).

${ }^{17}$ M. P. Gordon, Phys. Rev. A 8, 14 (1973).

${ }^{18}$ C. Hättig and B. A. Hess, J. Chem. Phys. 108, 3863 (1998).

${ }^{19}$ J. J. Molina, S. Lectez, S. Tazi, M. Salanne, J.-F. Dufrêche, J. Roques, E. Simoni, P. A. Madden, and P. Turq, J. Chem. Phys. 134, 014511 (2011).

${ }^{20}$ Y. Lauw, M. D. Horne, T. Rodopoulos, and F. A. M. Leermakers, Phys. Rev. Lett. 103, 117801 (2009). 